Ann. Biol. anim. Bioch. Biophys., Ig62, 2 (4), 319-328.

\title{
BASES MATHÉMATIQUES D'UNE INTERPRÉTATION ÉVENTUELLE DES SÉPARATIONS ÉLECTROPHORÉTIQUES INCOMPLÈTES.
}

\author{
Cl. LABOUCHE
}

Institut d'Élevage et de Médecine vétérinaire des Pays tropicaux, Laboratoire national do Recherches vétérinaires, Dakar (Sénégal)

\section{SOMMAIRE}

Les bases mathématiques d'une méthode permettant de déterminer le tracé et la surface d'une courbe de Gauss, connaissant les coordonnées de deux points autres que le sommet, sont exposées. Cette méthode pourrait éventuellement servir à l'interprétation des séparations électrophorétiques incomplètes.

La technique électrophorétique ne sépare qu'imparfaitement. les constituants protéiques du sérum des Ruminants domestiques, principalement ceux des Pays tropicaux (LABouche, I962 b, Rapp. Lab. Natl Rech. vét. Dakar, I959/60). Dans ces conditions, l'interprétation quantitative des électrophorégrammes peut conduire à des valeurs erronées (LABOUCHE, I $962 b$ ), le tracé densitométrique s'éloignant des limites réelles des courbes de Gauss représentatives des différentes fractions. Cet incident fâcheux se produisant souvent au niveau des sommets, la méthode mathématique que nous avons précédemment proposée (LABOUCHE, Ig62 a) devient inutilisable puisqu'elle est basée, en particulier, sur la mesure directe de la hauteur des pics.

Nous avons donc cherché à modifier cette méthode en utilisant des coordonnées de deux points quelconques, choisis sur des portions non perturbées des diagrammes. Il deviendrait en effet possible, dans ces conditions, de reconstituer les tracés réels des différentes fractions et d'en évaluer les concentrations relatives.

Le but de cette note est de préciser les bases mathématiques d'une interprétation éventuelle de ces séparations électrophorétiques insuffisantes. 


\section{Io Calcul des COURbes de Gauss a l'Aide des CoORdonnéEs DE DEUX POINTS AUTRES QUE LE SOMMET}

On sait que la formule des courbes de Gauss est :

$$
y=\frac{k n}{\sqrt{2 \pi}} \mathrm{e}^{-n^{2} x^{2} / 2 k^{2}} .
$$

La signification des paramètres $k$ et $n$ a été précisée dans un travail antérieur (LABOUCHE, I962 a). Leur connaissance suffit à définir la courbe et permet ainsi le calcul de l'ordonnée de tout point dont l'abscisse est connue.

Le problème revient donc à calculer $k$ et $n$ connaissant deux points d'ordonnées $\mathrm{U}_{1}$ et $\mathrm{U}_{2}$ et d'abscisse $Z_{1}$ et $Z_{2}$. Pour ce faire on peut tirer $k$ et $n$ de leur produit $k n$ et de leur rapport $k / n$.

\section{a) Calcul de $\mathrm{n} / \mathrm{k}$}

On peut écrire, en application de (I) :

$$
\begin{gathered}
\mathrm{U}_{1}=\frac{k n}{\sqrt{2 \pi}} \mathrm{e}^{-n^{2} \mathrm{Z}_{1}^{2} / 2 k^{2}} \quad \mathrm{U}_{2}=\frac{k n}{\sqrt{2 \pi}}=\mathrm{e}^{-n^{2} \mathrm{Z}_{2}^{2} / 2 k^{2}} \\
\frac{\mathrm{U}_{1}}{\mathrm{U}_{2}}=\frac{\mathrm{e}^{-n^{2} Z_{1}^{2} / 2 k^{2}}}{\mathrm{e}^{-n^{2} Z_{2}^{2} / 2 k^{2}}}=\mathrm{e}^{\left(n^{2} / k^{2}\right)\left[\left(Z_{2}^{2}-Z_{1}^{2}\right) / 2\right]}
\end{gathered}
$$

expression dont la forme logarithmique conduit à :

d'où :

$$
\log \mathrm{U}_{1}-\operatorname{L} \operatorname{og} \mathrm{U}_{2}=\frac{n^{2}}{k^{2}} \cdot \frac{Z_{2}^{2}-Z_{1}^{2}}{2}
$$

$$
n=\sqrt{\frac{2\left(\log U_{1}-\log U_{2}\right)}{Z_{2}^{2}-Z_{1}^{2}}} .
$$

b) Calcul de $\mathrm{kn}$

En prenant la forme logarithmique de (I) et en y remplaçant $\frac{I}{\sqrt{2 \pi}}$ par $y_{s}$ (ordonnée du sommet de la courbe normale), on peut écrire :

$$
\begin{gathered}
\mathrm{U}_{1}=k n y_{s} \mathrm{e}^{-n^{2} Z_{1}^{2} / 2 k^{2}} \\
\log \mathrm{U}_{1}=\log k n+\log y_{s}-\frac{n^{2} Z_{1}^{2}}{2 k^{2}}
\end{gathered}
$$

ou en remplaçant $n / k$ par (II) :

d'où :

$$
\log \mathrm{U}_{1}=\log k n+\log y_{s}-\frac{\log U_{1}-\log U_{2}}{Z_{2}^{2}-Z_{1}^{2}} Z_{1}^{2}
$$

$$
\begin{gathered}
\log k n=\log \frac{\mathrm{U}_{1}}{y_{s}}+\frac{Z_{1}^{2}}{Z_{2}^{2}-Z_{1}^{2}} \operatorname{L} \circ \frac{\mathrm{U}_{1}}{\mathrm{U}_{2}} \\
k n=\frac{\mathrm{U}_{1}}{y_{s}} \cdot\left(\frac{\mathrm{U}_{1}}{\mathrm{U}_{2}}\right)^{\mathrm{Z}_{1}^{2} /\left(\mathrm{Z}_{2}^{2}-\mathrm{Z}_{1}^{2}\right)}
\end{gathered}
$$


que 1'on peut encore écrire :

$$
k n=\frac{\mathrm{I}}{y_{s}} \frac{\mathrm{U}_{1}^{Z_{2}^{2} /\left(Z_{2}^{2}-Z_{1}^{2}\right)}}{\mathrm{U}_{2}^{Z_{1}^{2} /\left(Z_{2}^{2}-Z_{1}^{2}\right)}} \cdot
$$

c) Calcul de l'ordonnée $U$ d'un point quelconque

Compte tenu de (II) et de (III), (I) peut se mettre sous la forme :

$$
\mathrm{U}=\frac{\mathrm{U}_{\mathbf{1}}^{Z_{2}^{2} /\left(Z_{2}^{2}-Z_{1}^{2}\right)}}{\mathrm{U}_{\mathbf{2}} \mathrm{Z}_{1}^{2} /\left(\mathrm{Z}_{2}^{2}-Z_{1}^{2}\right)} \mathrm{e}^{\left.\left[\left(\log \mathrm{U}_{2}-\log \mathrm{U}_{1}\right) Z_{2}^{2}-Z_{1}^{2}\right)\right] Z^{2}}
$$

$Z$ étant l'abcisse du point dont l'ordonnée est $U$.

Ce qui peut encore s'écrire :

ou :

$$
\log U=\frac{Z_{2}^{2}}{Z_{2}^{2}-Z_{1}^{2}} \log U_{1}-\frac{Z_{1}^{2}}{Z_{2}^{2}-Z_{1}^{2}} \log U_{2}+\frac{\log U_{2}-\log U_{1}}{Z_{2}^{2}-Z_{1}^{2}} Z^{2}
$$

soit :

$$
\log U=\frac{x}{Z_{2}^{2}-Z_{1}^{2}}\left[\left(Z_{2}^{2}-Z^{2}\right) \cdot \log U_{1}+\left(Z^{2}-Z_{1}^{2}\right) \log U_{2}\right]
$$

$$
\mathrm{U}=\mathrm{U}_{1}\left(\mathrm{Z}_{2}^{2}-\mathrm{Z}^{2}\right) /\left(\mathrm{Z}_{2}^{2}-\mathrm{Z}_{1}^{2}\right) \mathrm{U}_{2}\left(\mathrm{Z}^{2}-\mathrm{Z}_{1}^{2}\right) /\left(\mathrm{Z}_{2}^{2}-\mathrm{Z}_{1}^{2}\right) .
$$

d) Calcul de la surface des courbes de Gauss

Nous avons déjà montré que la surface était égale à $k^{2} \cdot(\mathrm{LABOUCHE}, \mathrm{I} 962 a)$. Il suffira donc d'effectuer le produit :

$$
k n \times \frac{k}{n}
$$

pour obtenir la valeur de la surface.

\section{$2^{\circ}$ PRÉCISION DE LA MÉTHOdE}

a) Valeur de l'erreur dans le calcul des ordonnées

(V) peut se mettre sous la forme : $U=f_{\left(\mathrm{U}_{1}, \mathrm{u}_{2}, \mathrm{z}, \mathrm{z}_{\mathbf{2}}, \mathrm{z}_{1}\right)}$

$\mathrm{U}$ est donc une fonction de cinq variables indépendantes qui ne sont connues qu'avec une certaine approximation : $d \mathrm{U}_{1}, d \mathrm{U}_{2}, d \mathrm{Z}, d \mathrm{Z}_{2}, d \mathrm{Z}_{1}$.

$\mathrm{U}$ sera donc déterminé à une valeur $d \mathrm{U}$ près et l'on peut écrire :

$$
d \mathrm{U}=f^{\prime}{ }_{\left(\mathrm{U}_{1}\right)} d \mathrm{U}_{1}+f^{\prime}{ }_{\left(\mathrm{U}_{2}\right)} d \mathrm{U}_{2}+f^{\prime}(\mathrm{Z}) d \mathrm{Z}+f^{\prime}{ }_{\left(\mathrm{Z}_{1}\right)} d \mathrm{Z}_{1}+f^{\prime}{ }_{\left(\mathrm{Z}_{2}\right)} d \mathrm{Z}_{2}
$$

expression dans laquelle les termes $f^{\prime}$ représentent les dérivées de U par rapport à la variable correspondante rappelée en indice, les autres variables étant alors considérées comme constantes. 
L'erreur relative $d \mathrm{U} / \mathrm{U}$ sera obtenue en divisant chaque terme par U. Sans entrer dans le détail des calculs, indiquons simplement la valeur de chaque terme:

$$
\begin{aligned}
& f^{\prime}\left(\mathrm{I}_{1}\right) \frac{d \mathrm{U}_{1}}{\mathrm{U}}=\frac{\mathrm{Z}_{2}^{2}-\mathrm{Z}^{2}}{Z_{2}^{2}-\mathrm{Z}_{1}^{2}} \frac{d \mathrm{U}_{1}}{\mathrm{U}_{1}} \\
& f^{\prime}\left(\mathrm{U}_{2}\right) \frac{d \mathrm{U}_{2}}{\mathrm{U}}=\frac{Z^{2}-Z_{1}^{2}}{Z_{2}^{2}-Z_{1}^{2}} \frac{d \mathrm{U}_{2}}{\mathrm{U}_{2}} \\
& f^{\prime}\left(Z_{1}\right) \frac{d Z_{1}}{U}=\left\lfloor\frac{2 Z_{1}\left(Z_{2}^{2}-Z^{2}\right)}{\left(Z_{2}^{2}-Z_{1}^{2}\right)^{2}}\right]^{2}\left(\log U_{1}+\log U_{2}\right) d Z_{1} \\
& f^{\prime}\left(\bar{X}_{2}\right) \frac{d \mathrm{Z}_{2}}{\mathrm{U}}=\left[\frac{2 \mathrm{Z}_{2}\left(\mathrm{Z}_{1}^{2}-\mathrm{Z}^{2}\right)}{\left(\mathrm{Z}_{2}^{2}-\mathrm{Z}_{1}^{2}\right)^{2}}\right]^{2}\left(\log \mathrm{U}_{1}+\log \mathrm{U}_{2}\right) d \mathrm{Z}_{2} \\
& f^{\prime}(\mathrm{X}) \frac{d \mathrm{Z}}{\mathrm{U}}=\left[\left.\frac{2 \mathrm{Z}}{\mathrm{Z}_{2}^{2}-\mathrm{Z}_{1}^{2}}\right|^{2}\left(\log \mathrm{U}_{1}+\log \mathrm{U}_{2}\right) d \mathrm{Z}\right.
\end{aligned}
$$

L'erreur relative $d \mathrm{U} / \mathrm{U}$ est égale à leur somme. Celle-ci se simplifie dans quelques cas particuliers.

a. I) L'erreur ne porte que sur la mesure des ordonnées:

$$
\begin{gathered}
d \mathrm{Z}=d \mathrm{Z}_{1}=d \mathrm{Z}_{2}=\mathrm{o} \\
\frac{d \mathrm{U}}{\mathrm{U}}=\frac{\mathrm{Z}_{2}^{9}-\mathrm{Z}^{2}}{\mathrm{Z}_{2}^{2}-\mathrm{Z}_{1}^{2}} \frac{d \mathrm{U}_{1}}{\mathrm{U}_{1}}+\frac{\mathrm{Z}^{2}-\mathrm{Z}_{1}^{2}}{\mathrm{Z}_{2}^{2}-\mathrm{Z}_{1}^{2}} \frac{d \mathrm{U}_{2}}{\mathrm{U}_{2}}
\end{gathered}
$$

a. 2) Erreur relative dans la détermination de l'ordonnée du sommet, en absence d'erreur sur les abscisses.

$$
\begin{gathered}
\mathrm{Z}=d \mathrm{Z}=d \mathrm{Z}_{1}=d \mathrm{Z}_{2}=\mathrm{o} \\
\frac{d \mathrm{U}_{s}}{\mathrm{U}_{s}}=\frac{\mathrm{Z}_{2}^{2}}{\mathrm{Z}_{2}^{2}-\mathrm{Z}_{1}^{2}} \frac{d \mathrm{U}_{1}}{\mathrm{U}_{1}}+\frac{\mathrm{Z}_{1}^{2}}{\mathrm{Z}_{2}^{2}-\mathrm{Z}_{1}^{2}} \frac{d \mathrm{U}_{2}}{\mathrm{U}_{2}}
\end{gathered}
$$

Le terme en $\mathrm{U}_{2}$ devrait être affecté du signe $(-)$, mais comme on ne présage pas du signe des erreurs et que nous cherchons à exprimer une erreur maxima, nous avons utilisé le signe $(+)$.

Si on suppose qu'en valeur absolue, $d \mathrm{U}_{1}=d \mathrm{U}_{2}=\varepsilon$, on pourra écrire :

$$
\frac{d \mathrm{U}_{s}}{\mathrm{U}_{s}}=\frac{\varepsilon}{Z_{2}^{2}-Z_{1}^{2}}\left(\frac{Z_{2}^{2}}{\mathrm{U}_{1}}+\frac{Z_{1}^{2}}{\mathrm{U}_{2}}\right)
$$

On peut rendre cette expression plus générale en remplaçant $Z_{1}, U_{1}, Z_{2}, U_{2}$. par les coordonnées $\mathrm{X}_{1}, \mathrm{Y}_{1}, \mathrm{X}_{2}, \mathrm{Y}_{2}$ des points homologues de la courbe normale (LABOUCHE I962 a).

Rappelons ici les relations :

$$
Z=\frac{k \mathrm{X}}{n} ; \mathrm{U}=k n \mathrm{Y}
$$

Nous aurons alors :

$$
\frac{d \mathrm{U}_{s}}{\mathrm{U}_{s}}=\frac{\varepsilon}{k n\left(\overline{\mathrm{X}_{2}^{2}}-\mathrm{X}_{1}^{2}\right)}\left(\frac{\mathrm{X}_{2}^{2}}{\mathrm{Y}_{1}}+\frac{\mathrm{X}_{1}^{2}}{\mathrm{Y}_{2}}\right)
$$

Cette erreur relative a été évaluée dans un cas particulier : un des deux points servant au calcul est celui dont l'ordonnée est égale à la moitié de l'ordonnée du sommet, alors que le deuxième point prend des valeurs variables.

L'expression (VI) devient :

$$
\frac{d \mathrm{U}_{s}}{\mathrm{U}_{s}^{-}}=\frac{\varepsilon}{k n} \times \frac{\mathrm{I}}{\mathrm{X}_{2}^{2}-\mathrm{I}, 388} \cdot\left(\frac{\mathrm{X}_{2}^{2}}{\mathrm{O}, \mathrm{I} 99}+\frac{\mathrm{I}, 388}{\mathrm{Y}_{2}}\right) .
$$


L'erreur relative est proportionnelle à $\varepsilon$. Elle est d'autant plus faible que le sommet est plus élevé. Elle augmente considérablement pour $x_{2} \sim$ I, I7 8 et tend alors vers l'infini. Elle passe par un minimum pour $x_{2}=2$ puis augmente à nouveau légèrement.

Un examen plus poussé de (VI) montre que cette expression est le produit :

- d'un facteur indépendant de la forme de la courbe et qui ne fait intervenir que le choix des points de référence :

$$
\frac{I}{X_{2}^{2}-X_{1}^{2}}\left(\frac{X_{2}^{2}}{Y_{1}}+\frac{X_{1}^{2}}{Y_{2}}\right)
$$

- et d'un facteur dans lequel intervient la forme de la courbe $: \varepsilon / k n$.

L'expression (VII) : $\frac{d \mathrm{U}_{s}}{\mathrm{U}_{s}} \times \frac{k n}{\varepsilon}$ est donc constante quelle que soit la courbe considérée lorsque $X_{1}, X_{2}, Y_{1}, Y_{2}$ sont fixés.

De plus, il est aisé de montrer que $d \mathrm{U}_{s}$ ne dépend que de $\mathrm{X}_{1}, \mathrm{X}_{2}, \mathrm{Y}_{1}, \mathrm{Y}_{2}$. En effet :

$$
\frac{d \mathrm{U}_{s}}{\mathrm{U}_{s}} \times \frac{k n}{\varepsilon}=\frac{d \mathrm{U}_{s}}{k n \mathrm{Y}_{s}} \cdot \frac{k n}{\varepsilon}=\frac{d \mathrm{U}_{s}}{\varepsilon \mathrm{Y}_{s}}=\text { constante }
$$

$\mathrm{Y}_{s}$, sommet de la courbe normale est une constante ; $\varepsilon$ ayant été supposé constant, il en résulte que :

$$
d \mathrm{U}_{s}=\text { constante }
$$

quelle que soit la courbe considérée.

Les valeurs de (VII), $d \mathrm{U}_{s}$ ont été calculées pour $\mathrm{X}_{1}=\mathrm{I}, \mathrm{I} 78$ (soit $\mathrm{Y}_{1}=0, \mathrm{I} 99$ ) et pour $X_{1}=2\left(Y_{1}=0,054\right) ; X_{2}$ variant de 0,25 à 2,5 . Dans les deux cas, et à titre d'exemple, les tableaux ci-dessous donnent également la valeur de l'erreur relative $d \mathrm{U}_{s} / \mathrm{U}_{s}$ pour des courbes de Gauss dont on connaît $k n$ et $\mathrm{U}_{s}$. Ces courbes, de types

\begin{tabular}{|c|c|c|c|c|c|c|}
\hline \multicolumn{3}{|c|}{$X_{1}=1,178$} & \multicolumn{4}{|c|}{ Valeur de $\frac{d \mathrm{~L}_{s}}{\tau_{s}} \%$ pour des courbes de type: } \\
\hline $\mathrm{X}_{2}$ & $\frac{d \mathrm{~L}_{s}}{\mathrm{U}_{s}} \cdot \frac{k n}{\varepsilon}$ & $d \mathrm{U}_{s}, \mathrm{~mm}$ & albumine (a) & $\alpha(b)$ & $\beta(c)$ & $\gamma(d)$ \\
\hline 0,25 & $-\quad 2,9$ & $-\quad 0,6$ & $-\quad 0,6$ & - 2,4 & $-\quad 1,3$ & $-\quad 1,1$ \\
\hline 0,50 & $-\quad 4,6$ & $-\quad 0,9$ & $-\quad 0,9$ & $-\quad 3,6$ & $-\quad 1,9$ & $-\quad 1,6$ \\
\hline 0,75 & -9 & $\begin{array}{l}1,7 \\
\end{array}$ & $-1,8$ & - 7,2 & $-\quad 3,9$ & $-\quad 3,2$ \\
\hline 1,00 & $-27,7$ & $-\quad 5,5$ & $-5,7$ & $-22,8$ & $-1,2,2$ & $-10,3$ \\
\hline 1,178 & $-\infty$ & $-\infty$ & $-\infty$ & $-\infty$ & $-\infty$ & $-\infty$ \\
\hline & $+\infty$ & $+\infty$ & $+\infty$ & $+\infty$ & $+\infty$ & $+\infty$ \\
\hline 1,20 & +277 & +55 & +57 & +298 & $\begin{array}{l}+122 \\
+\quad 12\end{array}$ & $\begin{array}{l}+ \\
+103\end{array}$ \\
\hline 1,40 & $+33,4$ & $+6,7$ & $+6,9$ & +28 & $+14,8$ & $+12,4$ \\
\hline 1,60 & $+21,6$ & $+4, t^{\prime}$ & $+4,5$ & $+18,2$ & $\begin{array}{r}+9,7 \\
\end{array}$ & $\begin{array}{r}+8,1 \\
\end{array}$ \\
\hline 1,80 & $+18,3$ & $+3,7$ & $+3,8$ & $+15,4$ & $+8,2$ & $\begin{array}{r}\quad 6,8 \\
\end{array}$ \\
\hline 2,00 & $+17,5$ & $+3,5$ & $+3,6$ & $+14,6$ & $+7,5$ & $+6,5$ \\
\hline 2,20 & $+18,5$ & $+4,2$ & $\begin{array}{l}+4,3 \\
\end{array}$ & $+17,4$ & $\begin{array}{r}+9,2 \\
+\end{array}$ & $\begin{array}{r}7,7 \\
\end{array}$ \\
\hline \multirow[t]{2}{*}{2,50} & $+23,2$ & $+4,7$ & $+4,8$ & $+19,4$ & $+10,3$ & $+8,6$ \\
\hline & $\begin{array}{l}\text { a) } k n \\
\text { b) } k n\end{array}$ & $\begin{array}{l}\mathrm{U}_{s}, \mathrm{~m} \\
\mathrm{U}_{s}, \mathrm{~m}\end{array}$ & $\begin{array}{l}=97 \\
=24\end{array}$ & 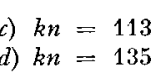 & $\begin{aligned} \mathrm{mm} & =45 \\
\mathrm{~nm} & =54\end{aligned}$ & \\
\hline
\end{tabular}
différents, ont déjà été utilisées dans un travail antérieur (LABouche I962 b) $\varepsilon$ est égal à $0,5 \mathrm{~mm}$. 
On voit ainsi que pour $\mathrm{X}_{1}=\mathrm{I}, \mathrm{I} 78, \mathrm{U}_{s}$ n'est connu avec une précision inférieure ou égale à $0,5 \mathrm{~mm}$ que pour $\mathrm{X}_{2}=0,25$ et jusqu'à $\mathrm{X}_{2}=0,75$ si on prend $\varepsilon=0, \mathrm{Imm}$. $\mathrm{Si}$ on choisit $\mathrm{X}_{1}=2, d \mathrm{U}_{s}$ n'est de l'ordre de $0,5 \mathrm{~mm}$ que pour $\mathrm{X}_{2}=0,25$ et jusqu'à $\mathrm{X}_{2}=\mathrm{I}, \mathrm{OO}-\mathrm{I}, 20$ pour $\varepsilon=0, \mathrm{I} \mathrm{mm}$.

\begin{tabular}{|c|c|c|c|c|c|c|}
\hline \multicolumn{3}{|c|}{$X_{1}=2,00$} & \multicolumn{4}{|c|}{ Valeur de $\frac{d \mathrm{U}_{s}}{\mathrm{U}_{s}}$ p. 100 pour des courbes de type : } \\
\hline$X_{2}$ & $\frac{d \mathrm{U}_{s}}{\mathrm{U}_{s}} \times \frac{k n}{\varepsilon}$ & $d \mathbf{U}_{s}, \mathrm{~mm}$ & albumine $(a)$ & $\alpha(b)$ & $\beta(c)$ & $\gamma(d)$ \\
\hline 0,25 & $-\quad 2,9$ & $-\quad 0,6$ & $-\quad 0,6$ & - 2,4 & 1,3 & $-1,1$ \\
\hline 0,50 & $\longrightarrow \quad 4,3$ & $-\quad 0,9$ & $-\quad 0,9$ & 3,6 & 1,9 & - \\
\hline 0,75 & $-\quad 6,9$ & $-\quad 1,3$ & $-\quad 1,4$ & 5,6 & 3,0 & - \\
\hline 1,00 & $-\quad 11,7$ & $-\quad 2,3$ & $-\quad 2,4$ & $-\quad 9,6$ & 4,9 & - \\
\hline 1,20 & $-18,5$ & $-\quad 3,7$ & $-\quad 3,8$ & $-\quad 15,2$ & $\begin{array}{l}-\quad 8,2 \\
\end{array}$ & $\begin{array}{l}-6,8 \\
\text { - }\end{array}$ \\
\hline 1,40 & $-\quad 30,9$ & $-6,1$ & $-6,3$ & $-\quad 25,2$ & $-13,5$ & $-11,3$ \\
\hline 1,60 & $-\quad 57,9$ & $-11,6$ & -12 & -48 & $-\quad 25,8$ & $-21,6$ \\
\hline 1,80 & $-145,6$ & $-29,1$ & -30 & -120 & - 64,5 & -54 \\
\hline & $-\infty$ & $-\infty$ & $一 \infty$ & $-\infty$ & $-\infty$ & $-\infty$ \\
\hline & $+\infty$ & $+\infty$ & $+\infty$ & $+\infty$ & $+\infty$ & $+\infty$ \\
\hline 2,2 & $+242,6$ & $+48,5$ & +50 & +200 & $+107,5$ & +90 \\
\hline $2, \overline{5}$ & +156 & +31 & +32 & +128 & $+\quad 69$ & +58 \\
\hline
\end{tabular}
a) $k n=22^{\prime} 3 \quad \mathrm{U}_{s}, \mathrm{~mm}=97$
b) $k n=60 \quad \mathrm{U}_{s}, \mathrm{~mm}=2^{\prime}$
c) $k n=113 \mathrm{U}_{s}, \mathrm{~mm}=45$
d) $k n=135 \quad \mathrm{U}_{s}, \mathrm{~mm}=5^{\prime} \mathrm{t}$

a. 3) Erreur relative dans la mesure de l'ordonnée d'un point quelconque, lorsque l'erreur de mesure ne porte que sur $U_{1}$ et $U_{2}$ et que $\varepsilon$ est identique pour $U_{1}$ et $U_{2}$.

La formule donnant l'erreur maxima est :

$$
\frac{d \mathrm{U}}{\mathrm{U}}=\frac{\varepsilon}{\mathrm{Z}_{2}^{2}-\mathrm{Z}_{1}^{2}}\left(\frac{Z_{2}^{2}-Z^{2}}{\mathrm{U}_{1}}+\frac{Z^{2}-Z_{1}^{2}}{\mathrm{U}_{2}}\right)
$$

dont la forme généralisée est :

$$
\frac{d \mathrm{U}}{\mathrm{U}}=\frac{\varepsilon}{k n} \times \frac{1}{\mathrm{X}_{2}^{2}-\mathrm{X}_{1}^{2}}\left(\frac{\mathrm{X}_{2}^{2}-\mathrm{X}^{2}}{\mathrm{Y}_{1}}+\frac{\mathrm{X}^{2}-\mathrm{X}_{1}^{2}}{\mathrm{Y}_{2}}\right)
$$

avec : $\mathrm{X} \neq \mathrm{X}_{1} \neq \mathrm{X}_{2}$.

Pour avoir une idée de l'erreur ainsi effectuée, nous avons calculé l'expression (VII) et $d \mathrm{U}$ dans les conditions suivantes :

$$
\begin{aligned}
& X_{1}=-I, I 78 ; Y_{1}=0, I 99 \\
& X_{2} \text { variant de } 0,25 \text { à } 2,50 \\
& X=I \text { et } X=2
\end{aligned}
$$

$d \mathrm{U} / \mathrm{U}$ est également indiqué pour une courbe de type albumine $(k n=243)$ et $\mathrm{E}=0,5 \mathrm{~mm}$. 


\begin{tabular}{|c|c|c|c|c|c|c|}
\hline \multirow[b]{2}{*}{$X_{2}$} & \multicolumn{3}{|c|}{$X=1$} & \multicolumn{3}{|c|}{$X=2$} \\
\hline & $\frac{d \mathrm{U}}{\mathrm{U}} \cdot \frac{k n}{\boldsymbol{\varepsilon}}$ & $d \mathrm{U}, \mathrm{mm}$ & $\frac{d \mathrm{U}}{\mathrm{U}}, \mathrm{p} \cdot 100$ & $\frac{d \mathrm{U}}{\mathrm{U}} \cdot \frac{k n}{\varepsilon}$ & $d \mathbf{U}, \mathrm{mm}$ & $\frac{d \mathrm{U}}{\mathrm{U}}, \mathrm{p} \cdot 100$ \\
\hline 0,25 & 4,21 & 0,5 & 0,9 & 9,83 & 0,26 & 2 \\
\hline 0,50 & 4,28 & 0,5 & 0,9 & 10,03 & 0,26 & 2 \\
\hline 0,75 & 4,22 & 0,5 & 0,9 & 10,40 & 0,27 & 2,1 \\
\hline 1,00 & - & - & - & 11,03 & 0,29 & 2,25 \\
\hline 1,10 & 4,06 & 0,5 & 0,8 & 11,46 & 0,31 & 2,4 \\
\hline 1,178 & - & - & - & - & - & - \\
\hline 1,20 & 4,06 & 0,5 & 0,8 & 50 & 1,3 & 10,3 \\
\hline 1,30 & 3,97 & 0,5 & 0,8 & 12,11 & 0,32 & 2,5 \\
\hline 1,40 & 3,91 & 0,5 & 0,8 & 12,51 & 0,34 & 2,6 \\
\hline 1,50 & 3,82 & 0,45 & 0,8 & 13,10 & 0,35 & 2,7 \\
\hline 1,60 & 3,71 & 0,45 & 0,8 & 13,89 & 0,38 & 2,9 \\
\hline 1,70 & 3,57 & 0,45 & 0,7 & 14,79 & 0,39 & 3 \\
\hline 1,80 & 3,43 & 0,4 & 0,7 & 15,79 & 0,42 & 3,2 \\
\hline 1,90 & 3,26 & 0,4 & 0,7 & 18,52 & 0,45 & 3,5 \\
\hline 2,00 & 3,02 & 0,4 & 0,6 & - & - & - \\
\hline 2,20 & 2,38 & 0,3 & 0,5 & 22,84 & 0,61 & 4,7 \\
\hline 2,50 & 0,73 & 0,1 & 0,1 & 33,93 & 0,91 & 7 \\
\hline
\end{tabular}

On constate que l'erreut maxima absolue, $d \mathrm{U}$, n'excède pas l'erreur directe de mesure $\varepsilon=0,5$ et quelle est d'autant plus faible que le point considéré est plus éloigné du sommet de la courbe.

\section{b) Valeur de l'erreur dans le calcul des surfaces}

La surface est obtenue en faisant le produit de $k n$ par $k / n$. L'erreur relative portant sur la surface sera donc égale à la somme des erreurs relatives de chacun de ces facteurs.

$\mathrm{Si}$ on pose $k n=\mathrm{K}$ et $k / n=\mathrm{R}$, on peut montrer par la méthode de la différentielle totale que :

$$
\begin{gathered}
\frac{\Delta \mathrm{K}}{\overline{\mathrm{K}}}=\mathrm{Z}_{2}^{2} \frac{\mathrm{Z}_{2}^{2}}{-Z_{1}^{2}} \frac{d \mathrm{U}_{1}}{\mathrm{U}_{1}}+\frac{\mathrm{Z}_{1}^{2}}{\mathrm{Z}_{2}^{2}-\mathrm{Z}_{1}^{2}} \frac{d \mathrm{U}_{2}}{\mathrm{U}_{2}} \\
\frac{\Delta \mathrm{R}}{\mathrm{R}}=\frac{\mathrm{I}}{2} \mathrm{~L} \operatorname{Lg} \frac{\mathrm{U}_{1}}{\mathrm{U}_{2}}\left(\frac{d \mathrm{U}_{1}}{\mathrm{U}_{1} \log ^{2} \mathrm{U}_{1}}+\frac{d \mathrm{U}_{2}}{\mathrm{U}_{2} \log ^{2} \mathrm{U}_{2}}\right)
\end{gathered}
$$

en supposant, pour simplifier, que l'erreur ne porte que sur la mesure des ordonnées $\mathrm{U}_{1}, \mathrm{U}_{2}$, les abcisses correspondantes étant considérées comme connues avec précision.

En appelant $\Delta S / S$, l'erreur relative maxima portant sur la surface et en remplaçant $\mathrm{U}_{1}$ et $\mathrm{U}_{2}$ par les ordonnées des points correspondants de la courbe normale, ona ura :

$$
\begin{aligned}
\frac{\Delta \mathrm{S}}{\mathrm{S}}=\frac{\mathrm{I}}{k n}\left[\frac{\mathrm{I}}{\mathrm{X}_{2}^{2}-\mathrm{X}_{1}^{2}}\left(\frac{\mathrm{X}_{2}^{2}}{\mathrm{Y}_{1}} d \mathrm{U}_{1}+\frac{\mathrm{X}_{1}^{2}}{\mathrm{Y}_{2}} d \mathrm{U}_{2}\right)\right. \\
\left.+\frac{\mathrm{I}}{2} \log \frac{\mathrm{Y}_{1}}{\mathrm{Y}_{2}}\left(\frac{d \mathrm{U}_{1}}{\mathrm{Y}_{1} \log ^{2} k n \mathrm{Y}_{1}}+\frac{d \mathrm{U}_{2}}{\mathrm{Y}_{2} \log ^{2} k n \mathrm{Y}_{2}}\right)\right] .
\end{aligned}
$$

Si on suppose que $d \mathrm{U}_{1}=d \mathrm{U}_{2}=\varepsilon$ et en transformant les logarithmes naturels en logarithmes décimaux, il vient :

$$
\frac{\Delta \mathrm{S}}{\mathrm{S}}=\frac{\varepsilon}{k n}\left[\frac{\mathrm{I}}{\mathrm{X}_{2}^{2}-\mathrm{X}_{1}^{2}}\left(\frac{\mathrm{X}_{2}^{2}}{\mathrm{Y}_{1}}+\frac{\mathrm{X}_{1}^{2}}{\mathrm{Y}_{2}}\right)+0,22 \log \frac{\mathrm{Y}_{1}}{\mathrm{Y}_{2}}\left(\frac{\mathrm{I}}{\mathrm{Y}_{1} \log ^{2} k n \mathrm{Y}_{1}}+\frac{\mathrm{I}}{\mathrm{Y}_{2} \log ^{2}} \frac{k n \mathrm{Y}_{2}}{2}\right)\right] .
$$


Dans le cas particulier où $\mathrm{X}_{2}=0$ (un des deux points servant au calcul est alors le sommet et $Y_{1} \sim 0,4$ ) l'expression précédente devient :

$$
\frac{\Delta \mathrm{S}}{\mathrm{S}}=\frac{\varepsilon}{k n}\left[2,5+0,22 \log \frac{0,4}{\mathrm{Y}_{2}}\left(\frac{2,5}{\log ^{2} \mathrm{U}_{s}}+\frac{\mathrm{I}}{\mathrm{Y}_{2} \log ^{2}} \overline{k n \mathrm{Y}_{2}}\right)\right]
$$

Nous avons appliqué cette formule au cas de la courbe de type " albumine" caractérisée par : $k n=243 ; \mathrm{U}_{s}=97 \mathrm{~mm}$, et pour une rerreur de mesure $\varepsilon=0,5 \mathrm{~mm}$. Nous avons envisagé quelques points remarquables de cette courbe, qui correspondent à :

$$
\begin{array}{ll}
\mathrm{X}_{2}=\mathrm{I} & \mathrm{Y}_{2}=0,242 \\
\mathrm{X}_{2}=\mathrm{I}, \mathrm{I} 78 & \mathrm{Y}_{2}=0,2 \\
\mathrm{X}_{2}=2 & \mathrm{Y}_{2}=0,054
\end{array}
$$

Les valeurs correspondantes de $\Delta S / S$ sont successivement $0,5,0,5$ et I, Io p. Ioo. On voit ainsi que l'erreur relative étant proportionnelle à $\varepsilon$, une erreur absolue sur $\mathrm{U}_{s}$ de $5 \mathrm{~mm}$ dans les 2 premiers cas et de $2,5 \mathrm{~mm}$ dans le troisième, ne se traduit que par un $\Delta \mathrm{S} / \mathrm{S}$ de l'ordre de $5 \mathrm{p}$. roo. Or nous avons vu que dans la plupart des cas $d \mathrm{U}_{s}$ est inférieur à ces valeurs (voir tableaux précédents).

Si nous nous plaçons maintenant dans le cas moins favorable où $k n$ est plus faible, on voit que l'erreur $d \mathrm{U}_{s}$ permise sera de :

$$
\begin{aligned}
& 0,6 \text { à } \mathrm{I}, 25 \mathrm{~mm} \text { pour la courbe }(\alpha) \\
& \mathrm{I}, 20 \text { à } 2,5 \mathrm{~mm} \text { pour les courbes }(\beta) \text { et }(\gamma)
\end{aligned}
$$

\begin{tabular}{|c|c|c|c|c|c|}
\hline \multirow[b]{2}{*}{$x_{1}$} & \multirow[b]{2}{*}{$\mathrm{X}_{2}$} & \multicolumn{4}{|c|}{$100 \mathrm{dS} / \mathrm{S}$} \\
\hline & & albumine & $\alpha$ & $\beta$ & $\gamma$ \\
\hline 0 & 1 & 0,53 & 2,2 & 1,1 & 0.97 \\
\hline 0 & 1,178 & 0,55 & 2,4 & 1,2 & 1,00 \\
\hline 0 & 2 & 1,1 & 13,7 & 3,7 & 2,7 \\
\hline 1,178 & 0 & 0,55 & 2,4 & 1,\}_{t}$ & 1,00 \\
\hline 1,178 & 1 & 5,7 & 23 & 12 & 10,3 \\
\hline 1,178 & 2 & 4 & 22 & 9,5 & 7,7 \\
\hline
\end{tabular}

Nous avons vu qu'il est possible de rester en deçà de ces limites lorsqu'on calcule U. (voir tableaux précédents).

Le tableau suivant récapitule l'erreur relative de surface pour chacune de nos courbes compte tenu des valeurs de $\mathrm{X}_{1}$ et $\mathrm{X}_{2}$, pour $\varepsilon=0,5 \mathrm{~mm}$.

c) Valeur de l'erreur maxima dans le calcul des surfaces relatives

Dans la pratique de l'analyse électrophorétique, on accorde plus d'intérêt à la valeur $\mathbf{P}$ exprimant le rapport de la surface d'une fraction $\mathrm{S}_{f}$ à la surface totale de l'électrophorégramme, $\Sigma S_{f}$, qu'à la surface $S_{f}$ elle-même. 
Nous avons montré que si $d \mathrm{~S}_{\text {f }}$ est l'erreur commise sur la détermination de $\mathbf{S}_{f}$, et $d \mathrm{P} / \mathrm{P}$ l'erreur relative de $\mathrm{P}$, on a l'égalité (Labouche I962 b).

$$
\frac{d \mathrm{P}}{\mathrm{P}}=\frac{\mathrm{I}-\mathrm{P}}{\mathrm{P}+\mathrm{S} f / d \mathrm{~S} f} .
$$

Le tableau suivant donne les valeurs de $d \mathrm{P} / \mathrm{P}$ pour $\mathrm{X}_{1}=$ o ou $\mathrm{I}, \mathrm{I} 78, \mathrm{X}_{2}$ étant variable et l'erreur absolue de mesure des ordonnées $U_{1}$ et $U_{2}$ étant $\varepsilon=0,5 \mathrm{~mm}$.

\begin{tabular}{|c|c|c|c|c|c|}
\hline \multirow[b]{2}{*}{$\mathrm{X}_{2}$} & \multirow[b]{2}{*}{$x_{1}$} & \multirow[b]{2}{*}{ albumine } & \multicolumn{2}{|c|}{$100 \mathrm{dP} / \mathrm{P}$} & \multirow[b]{2}{*}{$\gamma$} \\
\hline & & & $\alpha$ & $\beta$ & \\
\hline $\begin{array}{l}1 \\
1,17 \times \\
2\end{array}$ & $\begin{array}{l}0 \\
0 \\
0\end{array}$ & $\begin{array}{l}0,38 \\
0,39 \\
0,8\end{array}$ & $\begin{array}{c}1, ! \\
2,05 \\
11,5\end{array}$ & $\begin{array}{l}0,9 \\
1,0 \\
3,0\end{array}$ & $\begin{array}{l}0,4 \\
0,4 \\
1,2\end{array}$ \\
\hline $\begin{array}{l}0 \\
1 \\
2\end{array}$ & $\begin{array}{l}1,178 \\
1,178 \\
1,178\end{array}$ & $\begin{array}{l}0,3 ! 1 \\
4,0 \\
2,8\end{array}$ & $\begin{array}{r}2,0 \\
19,1 \\
18,4\end{array}$ & $\begin{array}{l}1,0 \\
9,8 \\
7,8\end{array}$ & $\begin{array}{l}0,4 \\
4,5 \\
3,4\end{array}$ \\
\hline
\end{tabular}

On constate que dans la plupart des cas, l'erreur relative sur $\mathrm{P}$ reste dans des limites acceptables.

\section{CONCLUSION}

Il apparait possible de calculer la surface d'une courbe de Gauss connaissant les coordonnées de deux points autres que le sommet. En choisissant ces points d'une manière judicieuse et compte tenu de l'erreur susceptible d'intervenir dans la mesure des ordonnées, on peut apprécier cette surface avec une précision suffisante.

En principe, cette méthode devrait permettre l'exploitation mathématique des diagrammes correspondant à des séparations électrophorétiques incomplètes. Les conditions pratiques d'appréciation seront envisagées dans une note ultérieure.

$$
\text { Reçu pour publication en mai } 1962
$$

\section{SUMMARY}

MATHEMATICAL BASES FOR AN EVENTUAL INTERPRETATION OF INCOMPIETE ELECTROPHORETIC SEPARATIONS

The electrophoretic technique only imperfectly separates the serum protein constituents of domestic ruminants, especially those of tropical countries. Under these conditions a quantitative interpretation of the electrophoretic diagrams may lead to incorrect values, the densimetric curve moving beyond the actual limits of the representative Gauss curves of the different fractions. Since this phenomenon occurs frequently at the peaks of the curves, the mathematical method we previously suggested becomes unserviceable because it is based in particular on the direct measurement of the height of the peaks.

We have, in consequence, tried to modify the method by making use of the coordinates of any two points, chosen on the undisturbed sections of the diagrams. Under these conditions it would in fact be possible to re-plot the actual curves of the different fractions and to calculate from them the relative concentrations.

The object of this paper is to define the mathematical bases for an eventual interpretation of these incomplete electrophoretic separations. 
The method for calculating the Gauss curves with the help of the coordinates of two different points on the peak is described, as is the calculation for the surface.

The accuracy of the method is calculated (error in the estimation of the ordinates and of the surfaces).

By a judicious choice of the two points of reference the surface can be calculated with suffcient precision. Hence, in principle, it is possible to make an interpretation of incomplete electrophoretic separations.

\section{RÉFÉRENCES BIBLIOGRAPHIQUES}

LABOUChE Cl., 1962 a. Méthode mathématique d'interprétation quantitative des électrophorèses sur papier. Ann. Inst. Pasteur, Paris, 102, 56ז-566.

LABOUChe Cl., 1962 b. Calcul d'erreurs dans l'interprétation quantitative des électrophorèses de sérum des Bovidés domestiques. Ann. Biol. anim. Bioch. Biophys. (sous presse).

Rapport sur l'activité du Laboratoire national de Recherches vétérinaires de Dakar-IIann, I959-I960. 